\title{
THE PLACE OF PENETRATING KERATOPLASTY IN CORNEAL DISEASES
}

\section{Snejana V. Murgova, Chavdar B. Balabanov, Georgi Y. Yordanov ${ }^{1}$}

\author{
Eye Clinic \\ University Hospital "G. Stranski” \\ Pleven \\ ${ }^{1}$ Department of Ophthalmology and \\ General Medicine \\ Trakia University \\ Stara Zagora
}

\begin{abstract}
Summary
The aim of the study was to analyze the results from penetrating keratoplasties (PK) in patients with different corneal diseases. The retrospective study included 124 patients (130 eyes) who underwent penetrating keratoplasty at the Eye Clinic, Pleven, between 1990 and 2011. The patients were divided into four groups, according to the main indication for keratoplasty: 1 bullous keratopathy - pseudophakic and aphakic bullous keratopathy (PBK and $\mathrm{ABK}) ; 2$ - degeneration and dystrophy (including keratoconus); 3 - keratitis, scar, trauma; 4 - repeat keratoplasty. The main indications for penetrating keratoplasty included regraft $-27 \%$, PBK $22.62 \%$, ABK $-11.9 \%$ and keratoconus - $10.71 \%$, though the leading cause had been different at different periods of time. By the time of suture removal (8-9 months on the average) in the group of patients with bullous keratopathy, the percentages of clear grafts were as follows: $55.88 \%$ in the group of patients with bullous keratopathy, $97.44 \%$ in the group with corneal degeneration and dystrophies, and $60 \%$ in the group with keratitis, corneal scar and trauma. In patients with regrafts, For the whole period of the study, grafts failed in $61.54 \%$ of patients with one regraft, and in $50 \%$ of the cases with second and third regrafts. Penetrating keratoplasty proved a valuable method of treatment for a number of corneal diseases. The prognosis and outcome, however, depended on the pathology responsible for corneal blindness.
\end{abstract}

Key words: penetrating keratoplasty, regraft, corneal diseases

\section{Introduction}

Corneal diseases are the second cause for the treatment of blindness. According to WHO data, they account for $5.1 \%$ of the global causes for total blindness. A study in our country has demonstrated a $3 \%$ frequency of corneal blindness in patients with visual acuity below 0.05 . The only method to improve the visual acuity in these cases is keratoplasty - replacement of damaged corneal tissue with healthy one [1-3]. 
The term keratoplasty was introduced by Franz Reisinger in 1854. In 1906, Eduard Zirm performed the first successful keratoplasty on a patient blinded by a lime burn. Since then the procedure has developed and the success rate has been increasing due to advances in surgical instrumentation, including microscopy and suture, the establishment of eye banks and tissue preservation, and surgical training programs and techniques. As a result, the number of keratoplasties has increased and indications have changed [4].

According to the aim of keratoplasties, they may be grouped into four categories:

- optical - to improve vision in cases of bullous keratopathy, keratoconus, corneal degenerations and dystrophies, corneal scar, regraft

- tectonic - to restore globe integrity in cases extreme corneal thinning, perforation, traumatic tissue loss

- therapeutic - to remove tissue in progressive keratitis after failed conservative treatment

- cosmetic - to restore normal eye appearance.

The aim of the study was to analyze the results after penetrating keratoplasties (PK) in patients with different corneal diseases.

\section{Material and Methods}

This is a retrospective study of 124 patients (130 eyes) with penetrating keratoplasty performed by one surgeon between 1990 and 2011 at the Eye Clinic Pleven University Hospital. The data collected included age, sex, and clinical indication for transplantation. In cases of regrafts, the initial diagnosis were recorded. Indications for keratoplasty were devided into 4 groups:

- bullous keratopathy - pseudophakic and aphakic bullous keratopathy (PBK and ABK)

- degeneration and dystrophy (including keratoconus)

- keratitis, scar, trauma

- repeat keratoplasty.

Standard penetrating keratoplasty was performed in all cases, using manual trephines for donor and recipient cornea. The donor cornea was sutured to the recipient, using interrupted sutures, $10 / 0$ nylon. If necessary, additional extraction, anterior vitrectomy, iris suture, or synechotomy. Graft status, visual acuity and complications were followed up and documented.

\section{Statistical analysis}

The results were described using tables, graphs and numerical indicators of structure, frequency, averages, correlations, etc. Statistical significance was evaluated with parametric tests for hypothesis testing in normal distribution of cases (Student's $t$-test, and one-way ANOVA) and by nonparametric tests in non-normal distribution (Pearson ${ }^{2}$, Mann-Whitney, KruscalWallis H-tests). The Kaplan-Maier test was applied for a time-event modeling and comparison of data. The significance of the results, conclusions and findings were determined at $\mathrm{p}<0.05$. All analyses were performed using statistical software packages Statgraphics Plus 4.1 for Windows, SPSS 14 (Statistical Package for the Social Sciences) and EXCEL for Windows.

\section{Results}

Of the 124 transplanted patients, $75(60.48 \%)$ were males and $49(39.52 \%)$ were females. The average age of patients in the recipient group, expressed by the median value was 63.5 years (range 7-87 years). The mean overall follow-up period was $22.33 \pm 3.45$ months (range 0.5 to 240). Figure 1 and 2 show the distribution of indications for $\mathrm{PK}$.

Bullous keratopathy - PBK and ABK (Figure 3) were the second and third most common indication for PK, with a peak in 1995 for ABK and 1996-2000, and after 2006 - for PBK. This group included 58 patients with one keratoplasty, ten cases with one regraft and 2 - with three regrafts. The median value of patients' age was 67 years (range 29-83). In the early postoperative period, the graft was clear in $79.31 \%$ of them. By the time of suture removal (8-9 months on the average) the grafts were clear in $55.88 \%$ of the cases. In the late postoperative period, mainly patients with complaints were followed up. Figures 4 and 5 show the Kaplan-Meier graft survival rate for the first year and at the end of the study. The visual acuity was above $0: 1$ in $26.92 \%$ of the patients. 


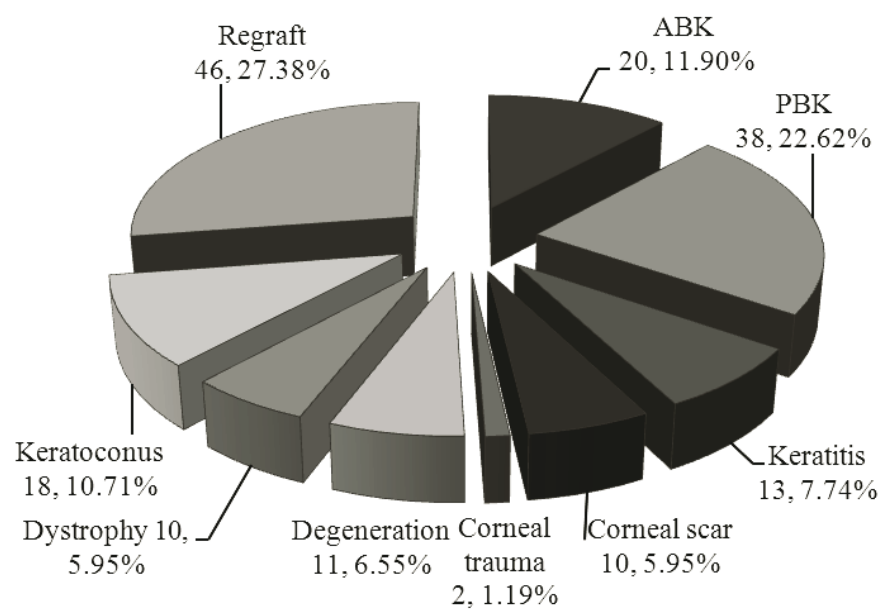

Figure 1. Indications for penetrating keratoplasty

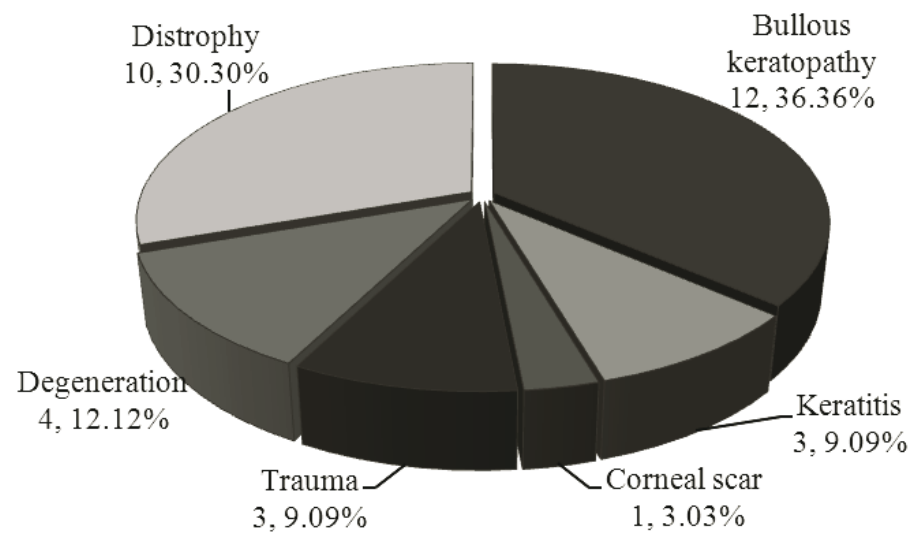

Figure 2. Initial indications for $\mathrm{PK}$ in patients with regrafts
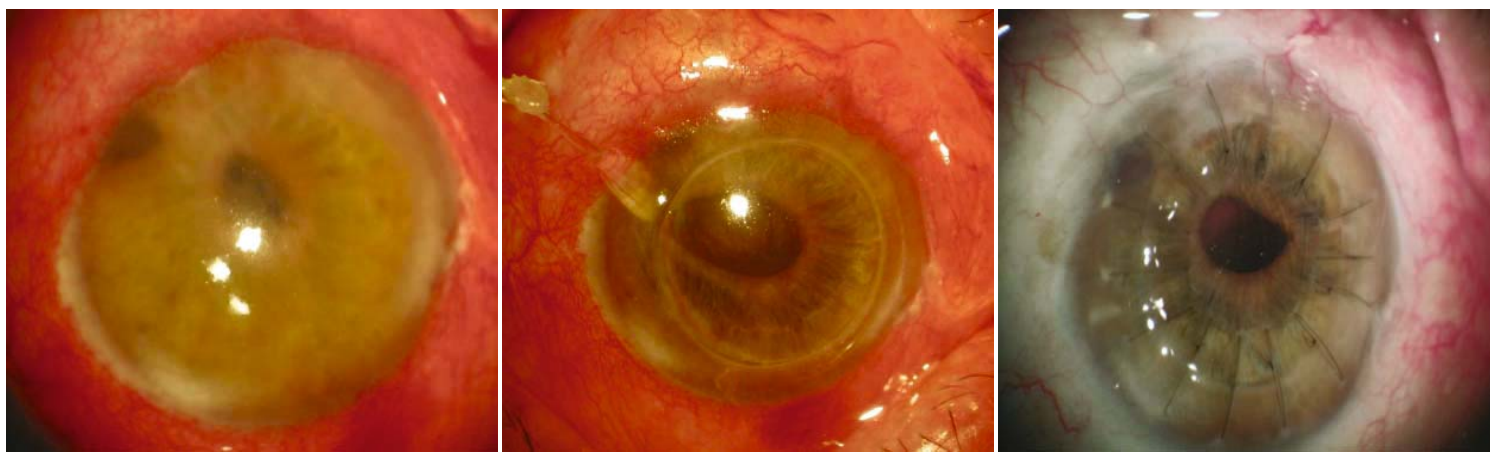

Figure 3. Keratoplasty with anterior vitrectomy in a patient with bullous keratopathy - preoperative and 5month postoperative outcome 


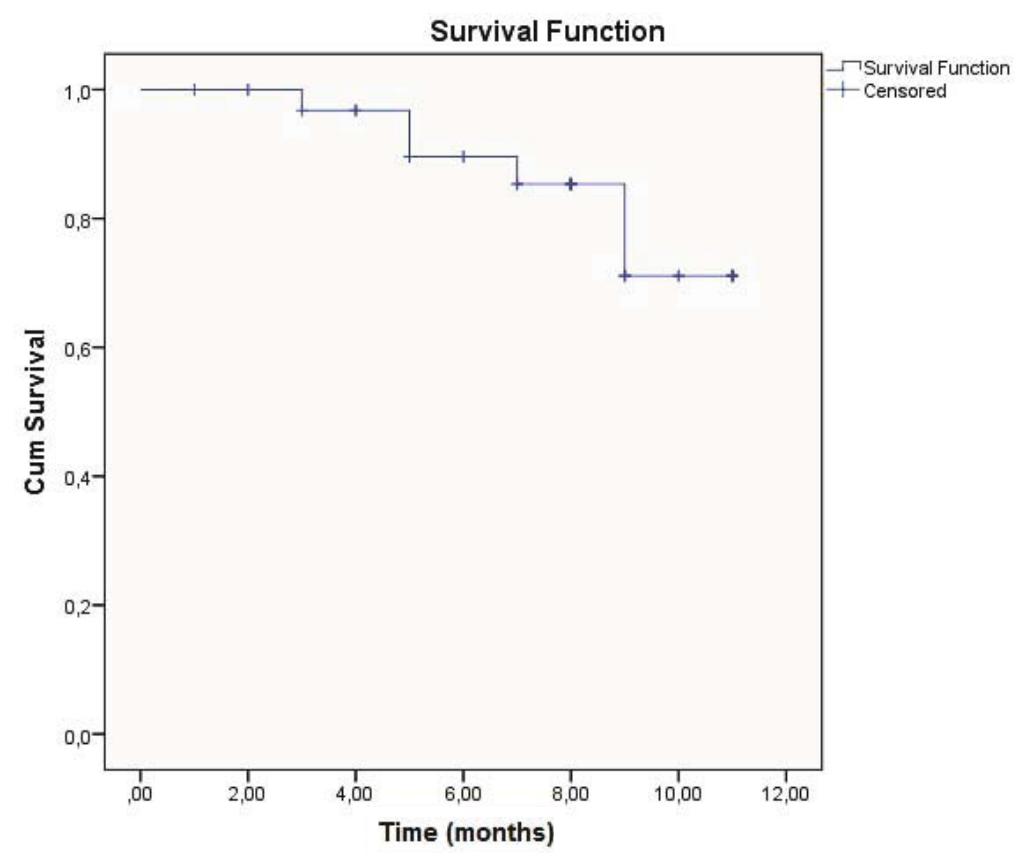

Figure 4. Kaplan-Meier graft survival rates in patients with bullous keratopathy for the first year

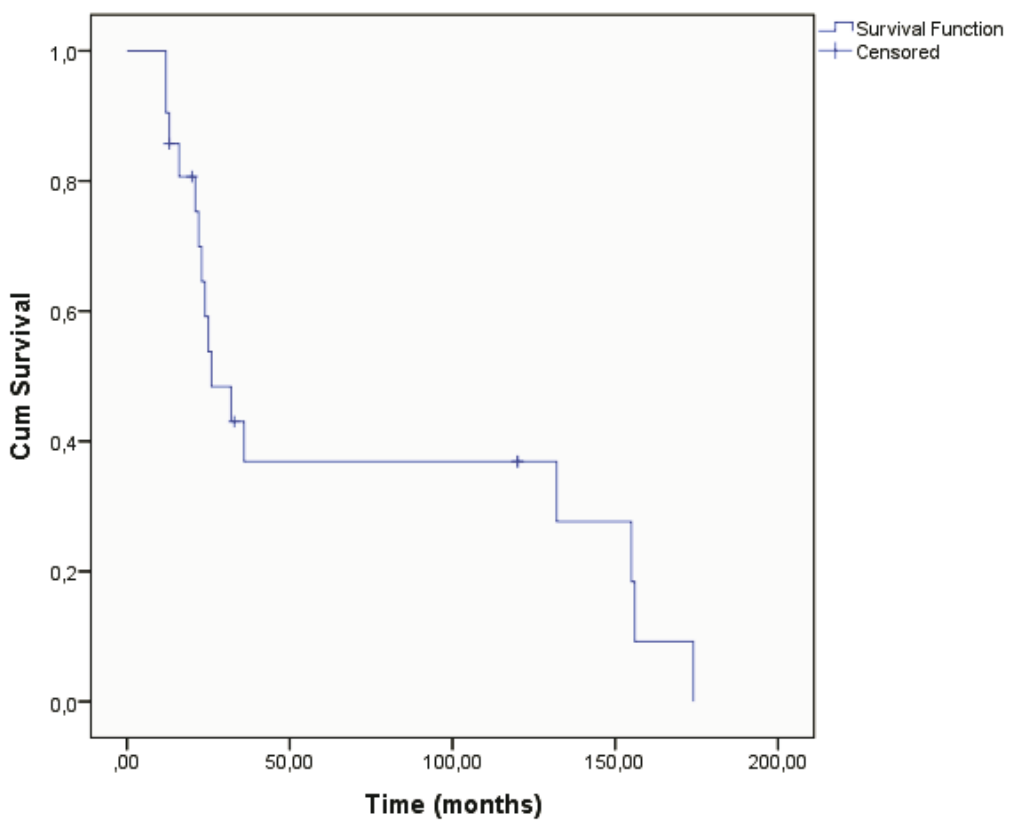

Figure 5. Kaplan-Meier graft survival rates in patients with bullous keratopathy at the end of the study.

The next group included patients with degeneration and dystrophies of the cornea (39 eyes), including patients with keratoconus, which accounted for $46.15 \%$ (Figure 6). The mean age of patients with degenerations and dystrophies on the cornea was $69.18 \pm 2.98$ years 32 keratoconus-30.5 years (median value; age range 15-72). During the first year, the graft failed in only one case with corneal degeneration. In that period, the indication for transplantation did not affect the survival rate $(\mathrm{p}=0.28)$ (Figure 7). After the first year, success rate for keratoconus was $89 \%$, and for other type of dystrophies and 
degenereation $-17 \%(p=0.01)$ (Figure 8). All patients had improvement of vision: of the patients with keratoconus, $44.44 \%$ had best corrected of visual acuity (BCVA) - above 0.4 , and for the rest of the group (19.05\%), the visual acuity was above 0.2 .
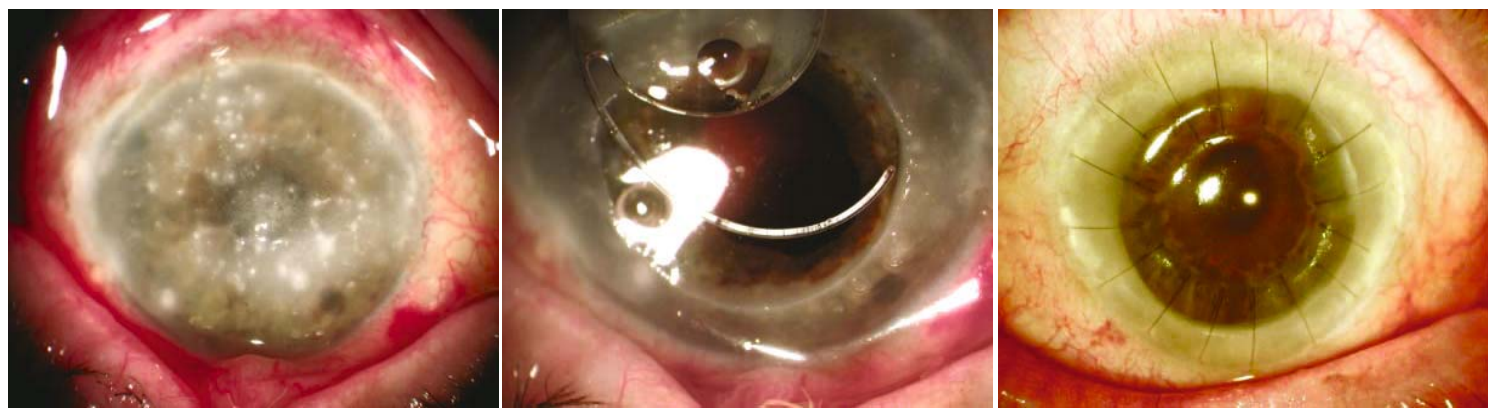

Figure 6. Keratoplasty and intraocular lens implantation in a patient with corneal degeneration - preoperative and postoperative outcome

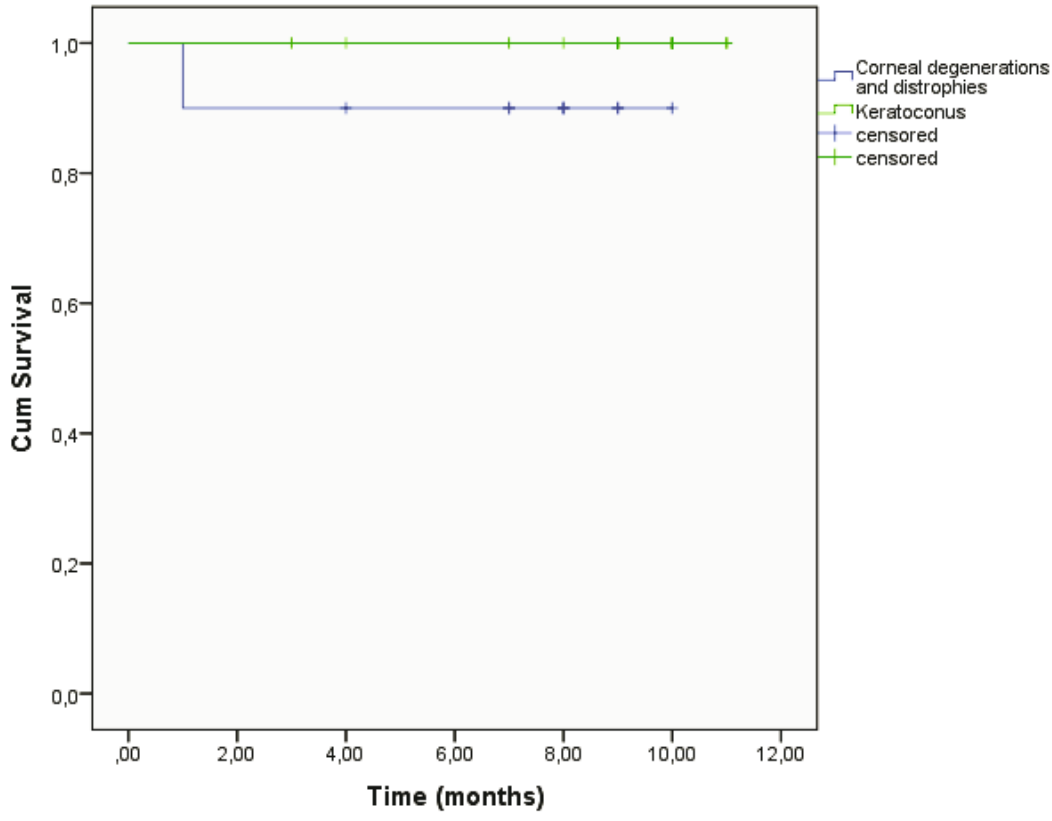

Figure 7. Kaplan-Meier graft survival rates in patients with degenerations and distrophies, including keratoconus for the first year.

Other less commonly seen indications keratits, corneal scar and trauma constituted $14.88 \%$ of all PK (Fig. 9). The average age for patients with keratitis was 64.61 years (range 1884 ), and for corneal scar $-53.7 \pm 3.62$ years (range $38-74)$. In the early postoperative period, only one graft in a patient with keratitis was opaque $(4 \%)$. At the end of the first year, $40 \%$ of grafts failed, mainly in cases with keratitis. The BCVA achieved was above 0.2 in $4.55 \%$ of the patients. 


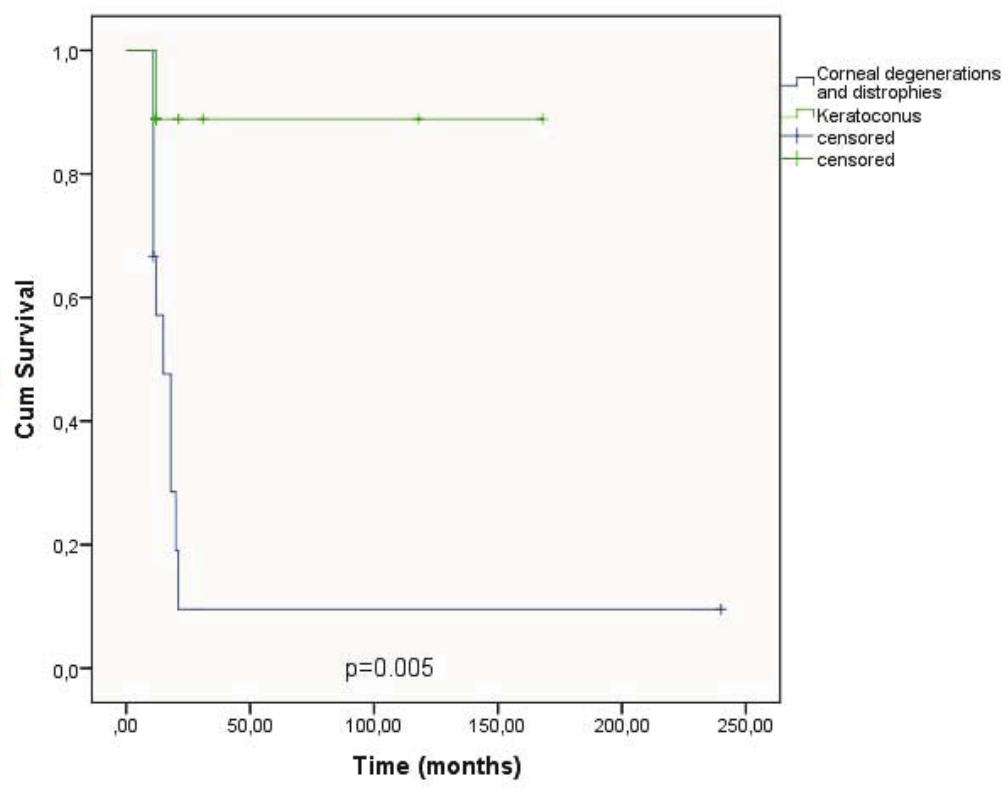

Figure 8. Kaplan-Meier graft survival rates in patients with degenerations and dystrophies, including keratoconus after the first year.
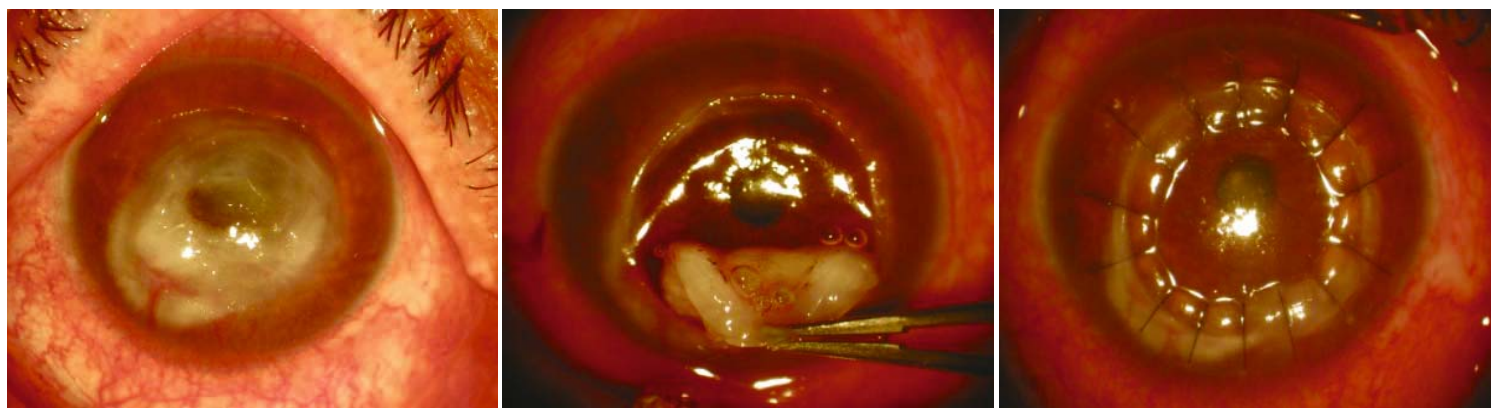

Figure 9. Keratoplasty in a patient with severe keratitis (descemetocele)

Regraft (Figure 10) was the leading indication for keratoplasty with a peak in 2000-2006. Of the 31 patients (33 eyes) with repeat keratoplasty, 10 had two and 5 had three regrafts. Figure 2 shows the initial indication for PK. The average time of

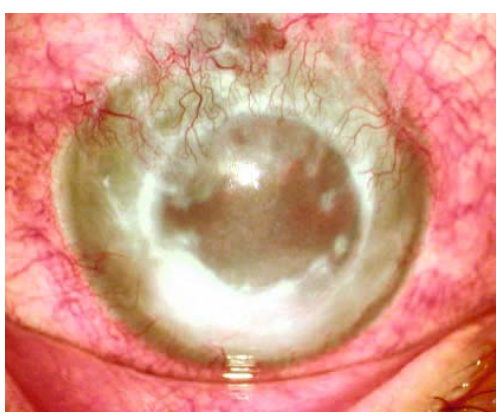

Figure 10. Patient with regraft - preoperative and postoperative outcome failure between the initial keratoplasty and first regraft was 19 months (range 1-180); between the first and second regraft the time of failure was 14 months (range 2-40); and between the second and third -27 months (range 0.5-73). In the early

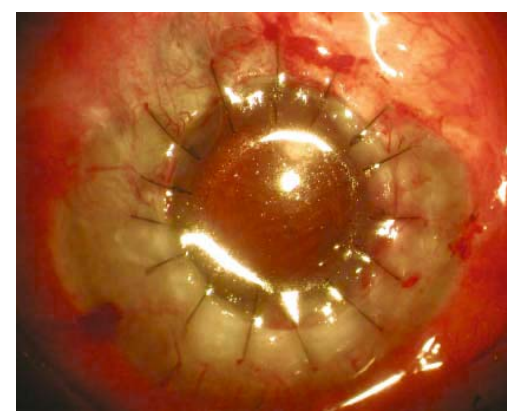


postoperative period (7 days after keratoplasty) the grafts were clear in $80-84 \%$ after the first, second and third keratoplasty. The Kaplan-Meier survival rate for the first year is presented on Figure 11. In the late postoperative period 27 patients with one regraft, 10 patients with two regrafts and 4 patients with three grafts were followed up. For the whole period of the study the grafts failed in $61.54 \%$ in patients with one regraft, and in 50\% - after the second and third regrafts. All patients had some improvement of vision. The commonest BCVA was between 0.05-0.09. The initial diagnoses and the number of keratoplasties did not affect the graft survival $(\mathrm{p}>0.05)$.

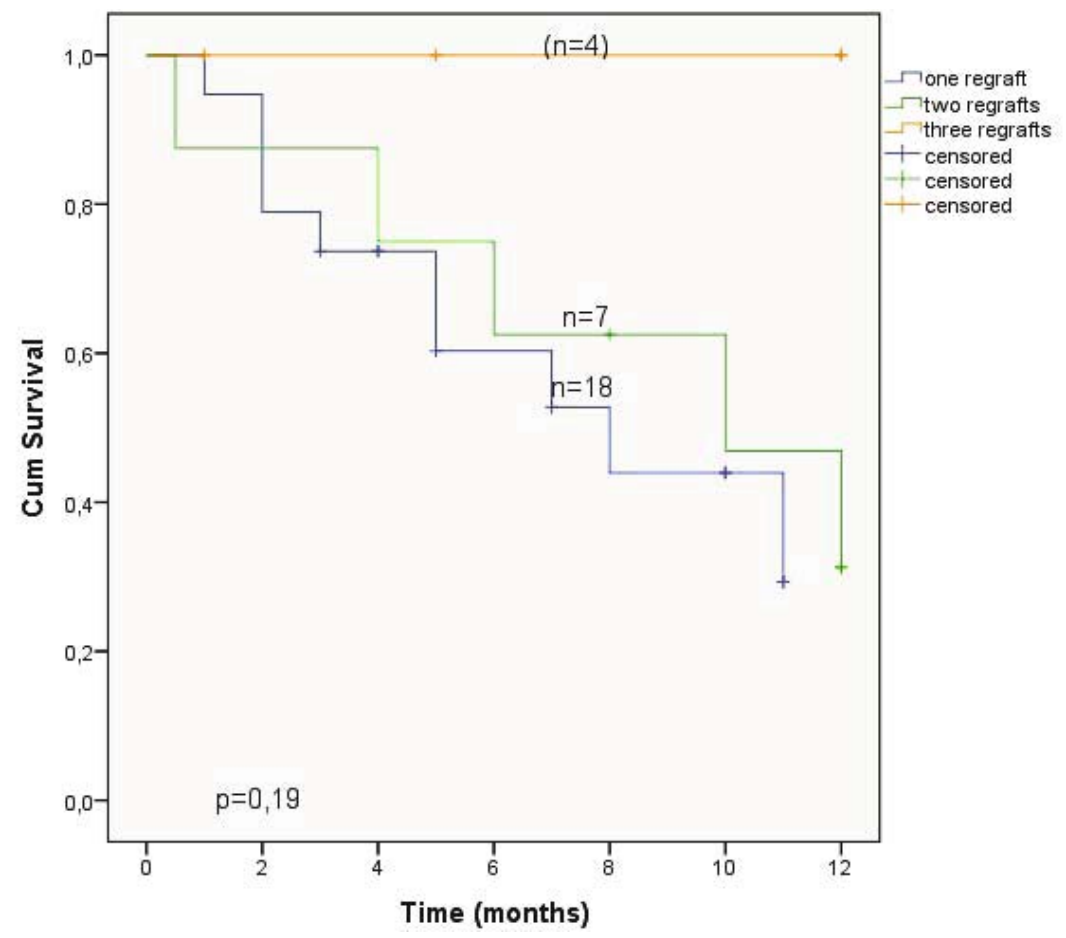

Figure 11. Kaplan-Meir survival graft rates in patients with regrafts

\section{Discussion}

The indications and results of $\mathrm{PK}$ at our institution during the period 1990-2011 were analyzed. Indications vary from country to country, and have changed over the years. According to our study, the main indications for PK were regraft - $27 \%$, followed by PBK $22.62 \%, \mathrm{ABK}-11.9 \%$ and keratoconus $10.71 \%$, but the leading indication was different at different periods of time. These indications have been recognized in the literature as the leading causes for PK worldwide [5-7].

Bullous keratopathy still remains the main indication for keratoplasty. The most common cause for corneal decompensation is cataract surgery. Before 1995 it was ABK, following intracapsular cataract extraction. The advent of intraocular lens implantation and introduction of new technologies in cataract surgery replaced the ABK with PBK. Despite the advancement in cataract surgery techniques, the use of viscoelastics and improved intraocular lens technology, the number of cases with PBK tends to increase. Multiple factors have been attributed to endothelial decompensation after cataract surgery - endothelial guttata, severe intraoperative trauma - especially that related to learning new surgical techniques, type of intraocular lens, postoperative glaucoma [8]. Patients' needs may be met using other methods of treatment - amnion, contact lens. However, keratoplasty provides both pain relief and vision improvement.

Keratoconus has been reported as number one indication for keratoplasty in some countries France, Germany, New Zealand, Zimbabwe, Israel. Resent reviews have demonstrated no 
male/female or ethnic group predominance [9]. The increasing interest in other methods - contact lenses, cross linking therapy, intracorneal rings may delay keratoplasty - lamellar or penetrating, based on the decision of the surgeon. These patients have the best prognosis concerning graft survival and visual acuity. The prognosis is not so good in cases of other types of dystrophy. In cases of deteriorated vision and pain, however, the method of choice is keratoplasty.

Keratoplasty may effectively treat severe keratitides, which cannot be controlled with medication. In cases of large corneal perforation $(>3 \mathrm{~mm})$, penetrating keratoplasty is the main choice of treatment. The aim is to restore the integrity of the eye and improve vision. Often, optical clarity in these cases cannot be achieved and in most of them a second graft can be planned. In patients with corneal scar, the only possibility to restore vision is keratoplasty.

Regraft numbers have increased, probably due to the increasing number of PK in patients who could potentially have graft failure, improvement of technical facilities, quality of treatment and greater experience in performing keratoplasty. In these patients we found, as have other studies in the literature, that BK (PBK and $\mathrm{ABK}$ ) is the most common indication for initial

\section{References}

1. Vasileva P. Problems of corneal transplantation in our country. Yearbook Euro Atlantic Transplant Alliance. 2006.p. 67-71. [In Bulgarian]

2. Whitcher JP, Srinivasan M, Upadhyay MP. Corneal blindness: a global perspective. Bulletin of the World Health Organization. 2001;79:21421.

3. Sinha R, Sharma N, Vajpayee RB. Corneal blindness - present status. Cataract \& Refractive Surgery Today. Oct 2005;59-61.

4. Mannis MJ, Krachmer JH. Keratoplasty: a historical perspective. Surv Ophthalmol. 1981;25(5):333-8.

5. Patel NP, Kim T, Rapuano CJ, Cohen EJ, Laibson PR. Indications for and outcomes of repeat penetrating keratoplasty, 1989-1995. Ophthalmology. 2000;107(4):719-24. keratoplasty. It is a result of a large number of keratoplasties in patients after cataract extraction. Regraft is a procedure with a high risk of rejection. Kirkness and coworkers have determined that graft failure is not related to the number of keratoplasties in the eye. It is the new corneal blood vessels that trigger the rejection [10]. In addition, it depends on the degree of damage of the recipient corneal tissue, advanced age, development of secondary glaucoma, surface disease etc. Despite the poor prognosis for such patients and the statistically increasing risk for each additional graft, keratoplasty is the only way to improve vision.

\section{Conclusion}

In conclusion, penetrating keratoplasty is a valuable method of treatment for a number of corneal diseases. It yields good results in patients with keratoconus and avascular corneal scars, and good therapeutic effect in corneal perforation with tissue loss. The improvement of medical management and the advent of more sophisticated surgical techniques in lamellar keratoplasty may change the indications for PK over the next years.

6. Edwards M, Clover GM, Brookes N, Pendergrast $\mathrm{D}$, Chaulk J, McGhee CN. Indications for corneal transplantation in New Zealand: 1991-1999. Cornea. 2002;21(2):152-5.

7. Yahalom C, Mechoulam H, Solomon A, Raiskup FD, Peer J, Frucht-Pery J. Forty years of changing indications in penetrating keratoplasty in Israel. Cornea, 2005;24(3):256-8.

8. Chen WL, Hu FR, Wang IJ. Changing Indications for penetrating keratoplasty inTaiwan from 1987 to 1999. Cornea. 2001;20(2):141-4.

9. Dorrepaal SJ, Cao KY, Slomovic AR. Indications for penetrating keratoplasty in a tertiary referral centre in Canada, 1996-2004. Can J Ophthalmol. 2007;42(2):244-50.

10. Kirkness CM, Ezra E, Rice NS, Steele AD. The success and survival of repeat corneal grafts. Eye (Lond). 1990;4(Pt 1):58-64. 